\title{
L'odeur du soi
}

\section{Les nouvelles de ce numéro ont été préparées par : Axel Kahn Jean-Claude Dreyfus Pascale Briand Marc Peschanski Jean-Pierre Grünfeld}

1. Thomas L. Fourth International Congress of Immunology. In : Netter E, Milgrom F, eds. Bâle : Karger, 1975 : 2-11.

2. Yamazaki, et al. Sensory distinction between $\mathrm{H}-2 \mathrm{~b}$ and $\mathrm{H}-2 \mathrm{bml}$ mutant mice. Proc Natl Acad Sci USA 1983 ; 80 : 5685-8.

3. Yamazaki, et al. Recognition of $\mathrm{H} 2$ types in relation to the blocking of pregnancy in mice. Science 1983 ; 221 : 186-8.

4. Barnes DM. Nervous and immune system disorders linked in a variety of diseases. Science $1986 ; 232$ : 160-1.

5. Angeletti RH, Hickey WF. A neuroendocrine marker in tissues of the immune system. Science $1985 ; 230: 89-90$.

$\mathrm{m} / \mathrm{s} n^{\circ} 1$ vol. 4 , janvier 88
Lorsqu'une souris a le choix de s'accoupler avec un partenaire consanguin ou avec une souris qui ne differe de la précédente que par son haplotype $\mathrm{H} 2$, elle manifeste un attrait particulier pour cette dernière - hommage à la différence ! Cette étrange observation fut faite il y a plus de dix ans et l'intérêt qu'on lui porte aujourd'hui résulte de la vision élargie qu'ont les immunologistes du complexe majeur d'histocompatibilité (CMH chez les vertébrés en général, $\mathrm{H} 2$ chez la souris, HLA chez l'homme). Ce complexe, à l'origine identifié par ses effets dans le rejet de greffes, intervient à de nombreux niveaux de la réponse immune : interactions entre les lymphocytes et les cellules présentant l'antigène, interactions entre différentes cellules lymphoïdes... Il aurait évolué, d'après Lewis Thomas, à partir d'un système qui avait pour fonction de maintenir l'intégrité des organismes grâce à un marquage chimique permettant de distinguer le " soi " de " l'autre " [1]. Un certain nombre de résultats viennent à l'appui de cette hypothèse. Des souris génétiquement identiques à l'exception de leurs haplotypes $\mathrm{H} 2$, reconnaissent les odeurs dégagées par leurs urines respectives, des fragments solubles de protéines codées par le complexe $\mathrm{H} 2$ pouvant à eux seuls, ou combinés à des produits du métabolisme, produire l'odeur identifiée par les souris [2]. Ainsi le complexe $\mathrm{H} 2$ peut à l'évidence conférer une spécificité olfactive, d'autres régions du génome, non encore identifiées, intervenant aussi dans la détermination de l'individualité olfactive des souris. Des résultats plus intéressants encore concernent la modification des comportements et des équilibres, en particulier hormonaux, qui résulte du type d'odeur perçue. Citons à nouveau l'accouplement préférentiel des souris d'haplotypes $\mathrm{H} 2$ différents évoqué en début d'article, mais aussi les modifications complexes qui conduisent à l'établissement d'une balance hormonale favorable ou non à l'implantation de l'embryon. Ainsi, une femelle successivement accouplée à un mâle puis, mis en présence d'un deuxième mâle (ou de ses urines), répond à l'odeur perçue par une balance hormonale favorable à l'implantation des embryons lorsque l'haplotype $\mathrm{H} 2$ du second mâle est identique à celui du géniteur. Il y a donc mémorisation de la première odeur, caractéristique de l'haplotype du géniteur, et établissement d'une balance hormonale particulière en réponse à la seconde stimulation olfactive, caractéristique de l'haplotype du second mâle introduit [3]. L'on conçoit bien qu'un tel système de communication chémosensoriel, capable d'influencer les mécanismes même de la reproduction, puisse être d'une importance capitale pour l'espèce qui le possède. Il est une facette supplémentaire des relations complexes qui unissent les systèmes immunitaire et nerveux [4], interactions dont certains agents commencent à être mis en évidence [5]. Nos affinités et nos répulsions sont-elles, comme dans le monde murin, en partie réglées par les lois olfactives de nos complexes HLA respectifs?

P.B. 